\title{
Catheter-Related vs. Catheter-Associated Blood Stream Infections in the Intensive Care Unit: Incidence, Microbiology, and Implications
}

\author{
Kristen C. Sihler, ${ }^{1}$ Carol Chenoweth,, ${ }^{2,3}$ Christy Zalewski, ${ }^{2}$ Wendy Wahl, ${ }^{1}$ Robert Hyzy, ${ }^{3}$ and Lena M. Napolitano ${ }^{1}$
}

\begin{abstract}
Background: Catheter-associated blood stream infections (CA-BSI) and catheter-related blood stream infections (CR-BSIs) differ in the degree of proof required to show that the catheter is the cause of the infection. The U.S. Centers for Disease Control and Prevention (CDC) National Healthcare Safety Network (NHSN; formerly the National Nosocomial Infections Surveillance [NNIS] group) collects data regarding CA-BSI nationally. We hypothesized that there would be a significant difference in the rates reported according to the definition.

Methods: Prospective surveillance of CA-BSI (defined as bacteremia with no extravascular source identified) is performed in all intensive care units (ICUs) at our institution and reported as the rate per 1,000 catheter-days. In January 2006, we initiated cultures of all catheter tips to evaluate for CR-BSI (defined as a catheter tip culture with $>15$ colony-forming units of the same microorganism(s) found in the blood culture) in the surgical, traumaburn, and medical ICUs.

Results: The CA-BSI rate across all ICUs for the 24-mo study period was 1.4/1,000 catheter-days. The CR-BSI rate was $0.4 / 1,000$ catheter days, for a rate difference of 1.0 infections $/ 1,000$ catheter-days $(\mathrm{p}<0.001 \mathrm{vs}$. CA-BSI). The pathogens identified in CA-BSI included many organisms that are not associated with catheter-related BSIs. Conclusions: The CR-BSI rate is significantly lower than the CA-BSI rate. The organisms identified in CA-BSI surveillance often are not common in catheter-related infections. Reporting CR-BSI thus is a more accurate measure of complications of central venous catheter use, and this rate may be more sensitive to catheter-specific interventions designed to reduce rates of BSI in the ICU.
\end{abstract}

C entral venous catheters (CVCs) are ubiquitous in intensive care units (ICUs). They are invaluable in providing venous access; a route for rapid, large-volume fluid resuscitation; a method of delivering medications that require central administration; and a tool for monitoring central venous pressure. However, they have been linked to blood stream infections (BSIs). It is estimated that 80,000 patients have catheter-associated BSIs (CA-BSIs) each year [1]. The mortality rate attributable directly to CA-BSIs is difficult to measure, and reports range from no attributable deaths [2] to a mortality rate as high as 35\% [3]. Each CA-BSI adds $\$ 11,971$ [4] to $\$ 40,890$ [5] to the hospital cost for a patient.

The U.S. Centers for Disease Control and Prevention (CDC) have issued evidence-based guidelines regarding insertion and maintenance of CVCs [6], and the implementation of the entire set of recommendations produces large and sustained reductions in the rates of attributable infections $[7,8]$. Substantial progress has been made in the reduction of CA-BSIs and CR-BSIs, but continued monitoring for CVC-related infections must still occur, as well as efforts to reduce the rate further.

The CDC defines a catheter-associated BSI (CA-BSI) as a BSI caused by an organism not related to another infection when a central line has been in place at some time during the $48 \mathrm{~h}$ prior to the collection of the blood culture. In contrast, a catheter-related BSI (CR-BSI) is defined as a BSI with either a positive catheter tip culture or a positive blood culture drawn from the CVC consistent with a culture drawn simultaneously from a peripheral site (Table 1) [9]. Whereas the first definition assumes the catheter is the default source of the infection if no

Departments of Surgery, ${ }^{1}$ Infection Control \& Epidemiology, ${ }^{2}$ and Internal Medicine, ${ }^{3}$ University of Michigan Health System, Ann Arbor, Michigan.

Presented in part at the $27^{\text {th }}$ Annual Meeting of the Surgical Infection Society, Toronto, Ontario, Canada, April 18-20, 2007. 


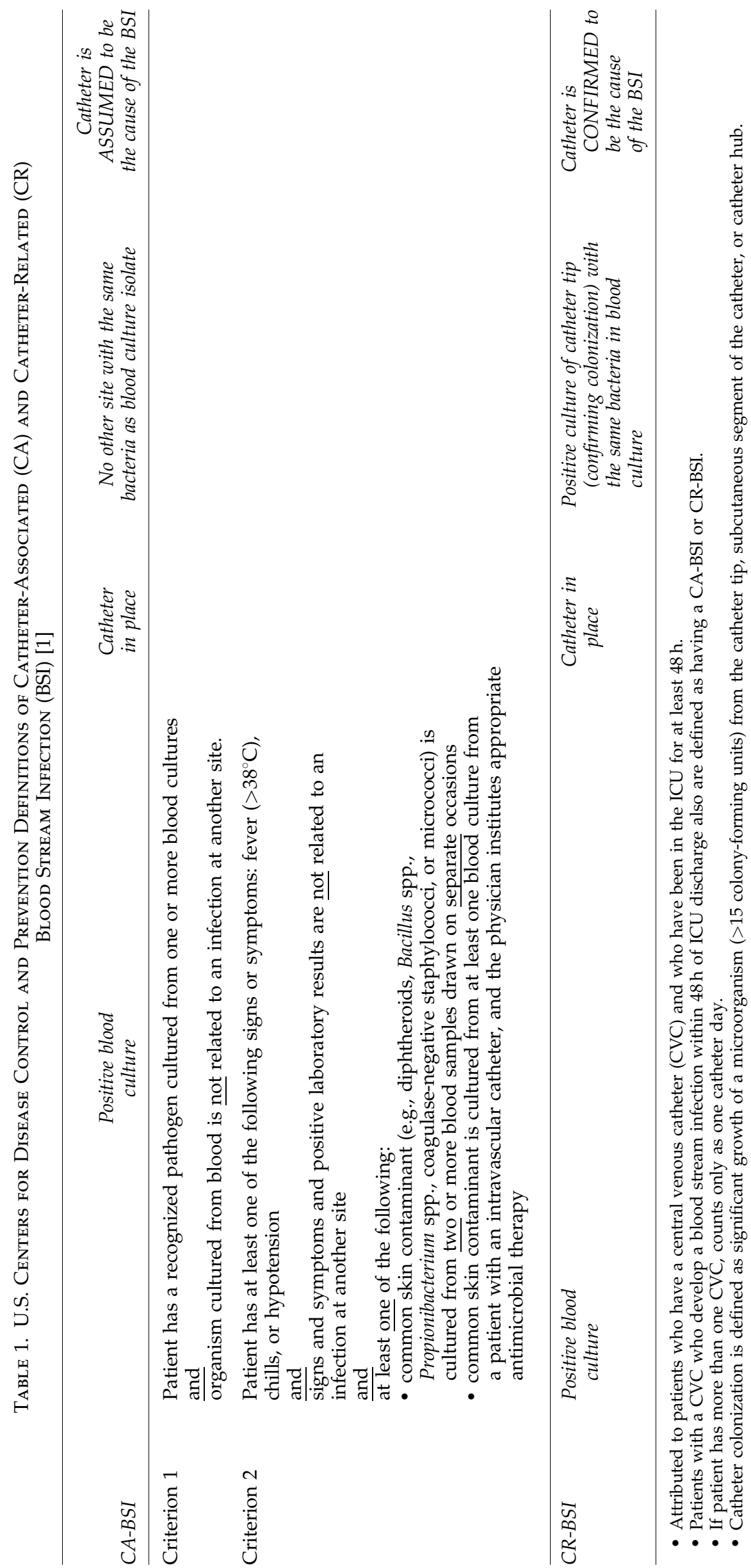


other site is found, the second requires proof of the involvement of the catheter in the infection. We hypothesized that CR-BSI rates would be significantly lower than CA-BSI rates and that use of CA-BSIs for institutional and national surveillance will significantly overestimate CR-BSIs.

\section{Materials and Methods}

\section{Study design and data collection}

This was a retrospective analysis of prospectively collected data at the University of Michigan, a 913-bed tertiary-care university-affiliated teaching hospital. This study was approved by the University of Michigan Human Studies Committee, and because there were no effects on treatment, the requirement for informed consent was waived.

In January 2006, we initiated culturing all CVC tips on removal in the surgical (20 beds), trauma/burn (10 beds), and medical (20 beds) ICUs to monitor the rates of CR-BSI. The data presented here represent the first 24 mos under this new reporting system. The rates of CA-BSI and CR-BSI in these ICUs were then compared.

All of the ICUs use standardized evidence-based strategies for the prevention of CR-BSI, including CVC placement from a cart with all necessary supplies, handwashing, chlorhexidine site preparation, full barrier precautions during insertion, halting the procedure if sterile technique is violated, and twice-daily inquiry regarding the possibility of removal of the CVC [8]. Each ICU has a dedicated infection control practitioner for monitoring of healthcare-associated infections. Prospective surveillance for CA-BSIs is performed according to the standards of the CDC National Healthcare Safety Network (NHSN; formerly National Nosocomial Infections Surveillance [NNIS] group) in ICUs and is reported as the rate per 1,000 catheter days. The rates of these infections are reported monthly to the institution's Infection Control Committee. Updates on CA-BSI and CR-BSI rates are reported monthly at the ICU quality improvement conference and are compared with both prior rates in the specific ICU and the published NHSN rates.

\section{Definitions}

A CA-BSI is defined as a BSI with a recognized pathogen that is not related to another infection. If a central line (defined as a vascular access device that terminates at or close to the heart or in one of the great vessels) was in use at any time during the $48 \mathrm{~h}$ prior to the onset of the BSI, the BSI is considered CA. A CR-BSI is defined as a BSI with a catheter tip yielding $\geq 15$ colony-forming units [CFU]) of the same organism found in a peripheral blood culture at the same time in addition to certain other clinical features (Table 1). Any CABSI that did not have a catheter tip and blood culture within $24 \mathrm{~h}$ of each other or did not have a catheter tip sent for culture at all was considered to be not "properly evaluated" for CRBSI. In these circumstances, CR-BSI was confirmed if a blood culture from the CVC was positive for the same organism as the peripheral blood culture, and no alternate source of secondary bacteremia was identified on clinical review.

\section{Results}

In the trauma/burn ICU, there were 13 CA-BSIs in 4,321 catheter days for a rate of 3.0/1,000 catheter days. Of these, two were CR-BSIs, for a rate of $0.5 / 1,000$ catheter days. In the surgical ICU, there were 10 CA-BSIs in 11,194 catheter days $(0.9 / 1,000$ catheter days). Of these, one was a CR-BSI $(0.09$ / 1,000 catheter days). In the medical ICU, there were $30 \mathrm{CA}$ BSIs during 11,886 catheter days ( $2.5 / 1,000$ catheter days). Of these, nine were documented CR-BSIs for a rate of $0.9 / 1,000$ catheter days (Table 2 and Fig. 1). However, two of the CABSIs in the surgical ICU and five in the trauma/burn ICU were not properly evaluated for CR-BSI, whereas all CA-BSIs in the medical ICU were properly evaluated. When proper evaluation for CR-BSI was not performed, we reviewed the patient record and confirmed both that the bacteremia was not related to the catheter and that there was an alternative cause for the secondary bacteremia. The common causes were confirmed to be pneumonia, abdominal infection, biliary infection, and severe skin and soft tissue infections. Of the seven CA-BSIs that were not properly evaluated, none was confirmed as a CR-BSI (Table 3).

Examination of the organisms isolated in the surgical ICU revealed that only one of the organisms from BSIs that were not properly evaluated has been reported as a cause of CRBSI: Candida parapsilosis [10]. The situation is different in the trauma/burn ICU, where all organisms isolated with the exception of Morganella morganii have been reported as causes of CA-BSI. The CR-BSIs in the medical ICU included four cases

Table 2. Rates of Catheter-Associated (CA) and Catheter-Related (CR) Blood Stream Infection (BSi) in Surgical ICU (SICU), Trauma-Burn ICU (TBICU), and Medical ICU (MICU)

\begin{tabular}{|c|c|c|c|c|c|c|c|}
\hline Site and year & $\begin{array}{c}\text { Central } \\
\text { venous } \\
\text { catheter days }\end{array}$ & Total BSIs ${ }^{\mathrm{a}}$ & Total BSI rate & $C A-B S I s$ & $C A-B S I$ rate & CR-BSIs & $C R$-BSI rate \\
\hline \multicolumn{8}{|l|}{ SICU } \\
\hline 2006 total & 5,799 & 5 & 0.9 & 5 & 0.9 & 0 & 0 \\
\hline 2007 total & 5,395 & 5 & 0.9 & 4 & 0.7 & 1 & 0.2 \\
\hline \multicolumn{8}{|l|}{ TBICU } \\
\hline 2006 total & 2,117 & 7 & 3.3 & 7 & 3.3 & 0 & 0 \\
\hline 2007 total & 2,204 & 5 & 2.3 & 3 & 1.4 & 2 & 0.9 \\
\hline \multicolumn{8}{|l|}{ MICU } \\
\hline 2006 total & 6,160 & 16 & 2.6 & 10 & 1.6 & 6 & 1.0 \\
\hline 2007 total & 5,726 & 12 & 2.1 & 9 & 1.6 & 3 & 0.5 \\
\hline
\end{tabular}

a"Total BSIs" includes both CA-BSIs and CR-BSIs. 


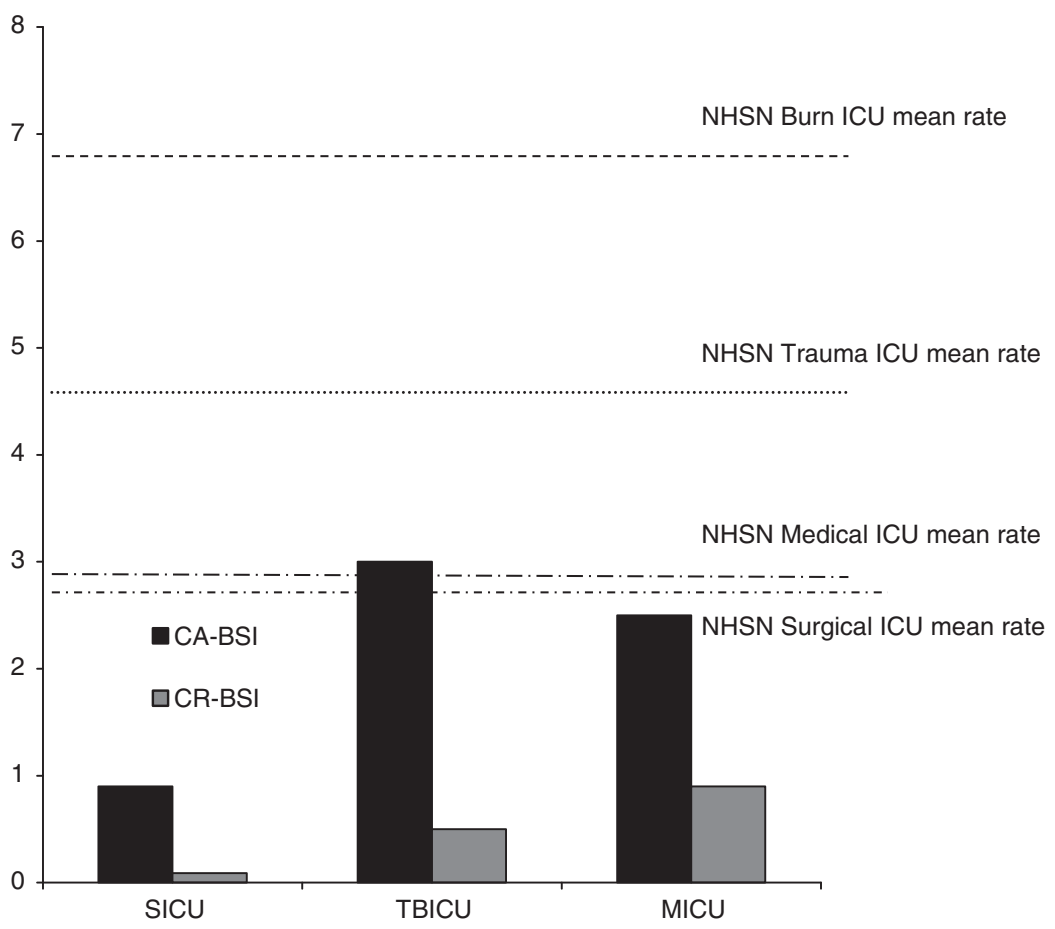

FIG. 1. Catheter-associated (CA) and catheter-related (CR) blood stream infection (BSI) rates in intensive care units (ICUs) over a two-year period (January 2006-December 2007) compared with the National Healthcare Safety Network national pooled mean rates (reported as rate of CA-BSI/1,000 catheter days) [12,13]. MICU=medical ICU; SICU = surgical $\mathrm{ICU}$; TBICU = trauma-burn ICU.

of vancomycin-resistant Enterococcus faecium, two cases of vancomycin-sensitive Enterococcus faecalis, two cases of coagulasenegative Staphylococcus, and one episode of methicillin-resistant Staphylococcus aureus (Table 3).

\section{Discussion}

Our ICUs, as with many ICUs across the country, have implemented measures to reduce the risk of CA-BSIs. However, most ICUs have not seen their infection rates decrease to zero. It is likely that many of the residual CA-BSIs are not caused by infection related to the CVC, and therefore, further changes in catheter-based interventions are unlikely to eliminate them.

Healthcare professionals should recognize the difference between surveillance and clinical definitions. The surveillance definitions for CA-BSI include all BSIs in patients with CVCs when other sites of infection have been excluded, and we fully recognize that some of the infections are secondary BSIs: They are not related to the CVC but are from undocumented sources (e.g., postoperative surgical site infection, intra-abdominal infection, pneumonia, or urinary tract infection). On the basis of the findings of this study, we recommend that the more rigorous definition of CR-BSI be used for surveillance, and that only BSIs for which other sources have been excluded and where a culture of the catheter tip demonstrates substantial colonies of an organism identical to those found in the blood stream be so defined.

The new publication of the CDC/NHSN surveillance definitions of healthcare-associated infections provides documentation that no significant changes have been made with regard to the CA-BSI definition for national surveillance [11]. A recently published report from the Evaluation of Processes and Indicators in Infection Control (EPIC) Study Group [12] acknowledged the substantial potential measurement errors in the collection of BSI rates in the hospital setting related to failure to apply the NHSN surveillance criteria accurately. Problems with the definitions [13] resulted in a likelihood that some secondary BSIs were included in the data analysis and that BSIs identified by a single positive blood culture may have represented contaminants rather than true infections [14].

Accurate measurement of the rates of BSIs arising from catheters is important because ICUs with high rates of CRBSIs will need to institute further performance improvement initiatives in CVC insertion and management. By contrast, ICUs that have high rates of CA-BSIs that are not CR-BSIs will find that changes in CVC-based interventions are ineffective. Although it is more costly to evaluate for CR-BSI because of the cost of catheter tip culture in addition to the cost of blood cultures, it is currently the only method to ensure that the catheter is the cause of the BSI, as endoluminal catheter colonization is invariably present in cases of CR-BSI. However, it should be recognized that the implementation of ineffective measures to reduce BSI rates related to an inaccurate definition is more costly yet. Additionally, focusing erroneously on the catheter as the source of the BSI may lead the clinician to overlook other potential causes of nosocomial BSIs.

Monitoring of BSIs secondary to CVCs is advocated by the CDC and NHSN. One of the goals of the NHSN is to evaluate interventions, and one of the expressly described uses of the NHSN database is to enable interhospital comparisons [15]. 


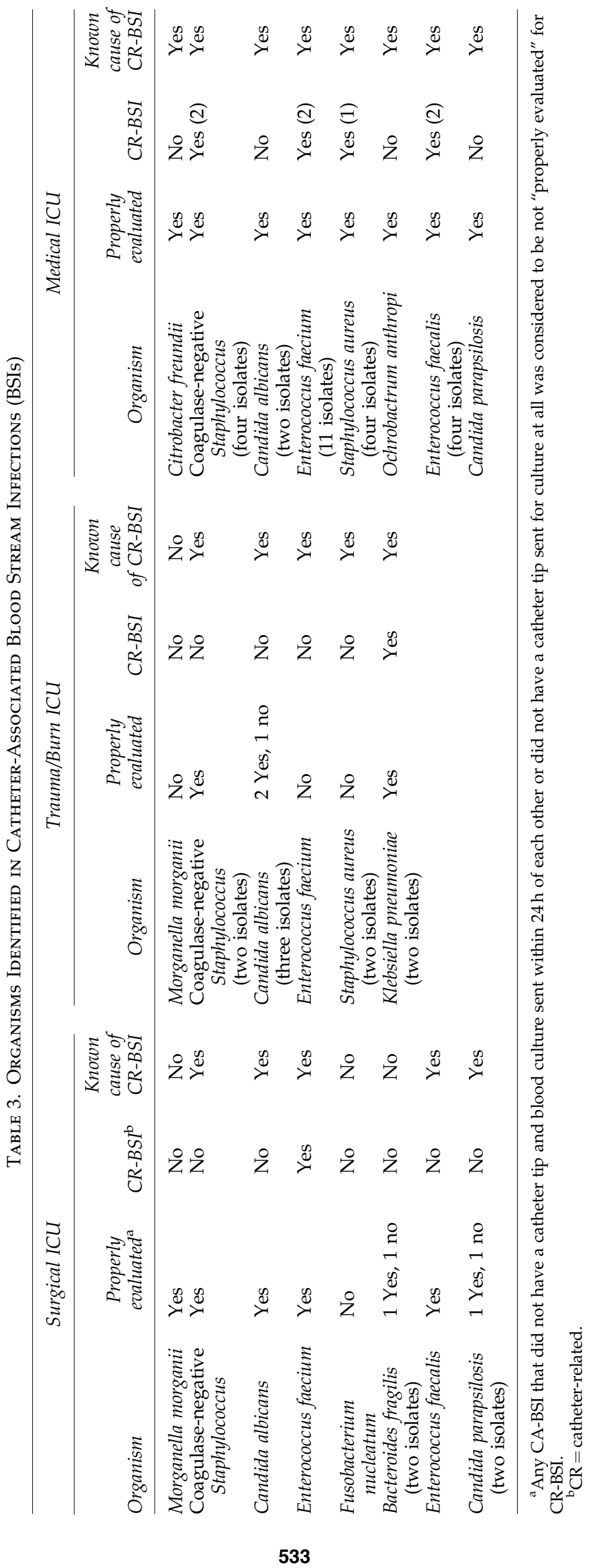


Data from the last two reports from the NNIS in 2004 and the NHSN in 2007 have already documented a significant reduction in CA-BSI in U.S. institutions that participate in this hospital-based surveillance program [12,13].

This surveillance is becoming even more important now that BSIs related to CVCs are one of the publicly reportable measures of health care quality being demanded by consumers and payors. Public reporting of adherence to best practices is already available in some areas, and Pennsylvania and 22 other states provide information to the public about individual hospitals' nosocomial infection rates, including BSIs.

In addition, starting October 1, 2008, Medicare will not pay the additional costs incurred by certain hospital-acquired infections or medical errors. After issuing a proposed set of measures and considering comments from stakeholders and experts, the Centers for Medicine and Medicaid Services (CMS) decided to disallow incremental payments associated with eight secondary conditions that it sees as preventable complications of medical care, including vascular catheterassociated infection. The final rule implements Congressional law Section 5001(c) of the Deficit Reduction Act of 2005 [16]. With this new rule, hospitals will no longer receive additional payment for these eight conditions identified by the CDC unless the condition was present at the time of admission. According to the CDC, health care-associated infections result in as much as $\$ 27.5$ billion in additional health care expenses annually [17]. A unique International Classification of Diseases, version 9, Clinical Manual (ICD-9-CM) code for vascular catheter-associated infection was introduced in fiscal year 2008 with the Inpatient Prospective Payment System (IPPS) proposed rule (new code for vascular catheter-associated infection, 999.31: Infection due to central venous catheter [18]), which became effective October 1, 2007 [19]. The use of CA-BSI for surveillance in hospitals will now have significant financial implications according to the results of this study that documented that CA-BSI overestimates true CR-BSI.

Healthcare practitioners are in agreement that elimination of healthcare-associated and device-associated infections is in the best interest of patients and that practitioners are obligated to provide the best care to achieve this result. Furthermore, it is crucially important that the correct definitions of healthcare-associated infections be utilized. In the data provided in this study, we have shown that CA-BSI is not specific for catheter infections and recommend that the CDC and the NHSN advocate the use of CR-BSI as the standard surveillance definition for central venous catheter-related BSI.

\section{Author Disclosure Statement}

No conflicting financial interests exist.

\section{References}

1. Mermel LA. Prevention of intravascular catheter-related infections. Ann Intern Med 2000;132:391-402.

2. Pelletier SJ, Crabtree TD, Gleason TG, et al. Bacteremia associated with central venous catheter infection is not an independent predictor of outcomes. J Am Coll Surg 2000;190: 671-680.

3. Pittet D, Tarara D, Wenzel RP. Nosocomial bloodstream infection in critically ill patients: Excess length of stay, extra costs, and attributable mortality. JAMA 1994;271:1598-1601.
4. Warren DK, Quadir WW, Hollenbeak CS, et al. Attributable cost of catheter-associated bloodstream infections among intensive care patients in a nonteaching hospital. Crit Care Med 2006;34:2084-2089.

5. Blot SI, Depuydt P, Annemans L, et al. Clinical and economic outcomes in critically ill patients with nosocomial catheter-related bloodstream infections. Clin Infect Dis 2005;41:1591-1598.

6. O'Grady NP, Alexander M, Dellinger EP, et al. Guidelines for the prevention of intravascular catheter-related infections. Centers for Disease Control and Prevention. MMWR Recomm Rep 2002;51(RR-10):1-29.

7. Berenholtz SM, Pronovost PJ, Lipsett PA, et al. Eliminating catheter-related bloodstream infections in the intensive care unit. Crit Care Med 2004;32:2014-2020.

8. Pronovost $\mathrm{P}, \mathrm{Needh}$ am $\mathrm{D}$, Berenholtz $\mathrm{S}$, et al. An intervention to decrease catheter-related bloodstream infections in the ICU. N Engl J Med 2006;355:2725-2732.

9. Garner JS, Jarvis WR, Emori TG, et al. CDC definitions for nosocomial infections, 1988. Am J Infect Control 1988;16: 128-140.

10. Inoue $Y$, Kohno S, Fujii T, et al. Clinical evaluation of catheterrelated fungemia and bacteremia. Intern Med 1995;34: 485-490.

11. Horan TC, Andrus M, Dudeck MA. CDC/NHSN surveillance definition of health care-associated infection and criteria for specific types of infections in the acute care setting. Am J Infect Control 2008;36:309-332.

12. Kritchevsky SB, Braun BI, Kusek L, et al. The impact of hospital practice on central venous catheter associated bloodstream infection rates at the patient and unit level: A multicenter study. Am J Med Qual 2008;23:24-38.

13. Mermel LA. Defining intravascular catheter-related infections: A plea for uniformity. Nutrition 1997;13(4 Suppl):2S-4S.

14. McDonald C, Carrico R, Simmons B, et al. (editors). The role of single positive blood cultures in the surveillance of bloodstream infections caused by common skin contaminants: An analysis of EPIC data. Presented at the Annual Meeting of the Society for Healthcare Epidemiology of America (SHEA). Toronto, Canada, 2001.

15. National Nosocomial Infections Surveillance System (NNIS). About NNIS 2005 [updated February 16, 2005 and May 24, 2007]. Available at www.cdc.gov/ncidod/dhqp/nnis.html

16. Services CfMM. Changes to the Hospital Inpatient Prospective Payment Systems and Fiscal Year 2008 Rates. [August 1, 2008]. Available at www.cms.hhs.gov/AcuteInpatientPPS/downloads/CMS-1533-FC.pdf

17. Lawrence D. Costly infections. A new CMS hospitalacquired infection mandate is putting hospitals on the alert. Healthcare Inform 2007;24:10, 2, 4.

18. Services CfMM. Updates and Revisions to the ICD-9-CM Diagnosis Codes. [August 1, 2008]. Available at www .cms.hhs.gov/ICD9ProviderDiagnosticCodes/Downloads/ new_diagnosis_codes_2007.pdf

Address correspondence to: Dr. Kristen C. Sihler

Division of Acute Care Surgery Department of Surgery University of Michigan 1500 E. Medical Center Dr.

Ann Arbor, MI 48109-0033

E-mail: ksihler@med.umich.edu 